\title{
PIRÓLISE DE RESÍDUO DE COURO CURTIDO AO CROMO EM REATOR DE LEITO FLUIDIZADO
}

\author{
T. SIMIONI ${ }^{1}$, A. DETMER ${ }^{2}$ \\ ${ }^{1}$ Universidade Federal de Santa Catarina, Departamento de Engenharia Química e \\ Engenharia de Alimentos \\ ${ }^{2}$ Universidade de Caxias do Sul, Centro de Ciências Exatas e Tecnologias \\ E-mail para contato: taysnarasimioni@gmail.com
}

\begin{abstract}
RESUMO - A indústria coureira gera uma grande quantidade de resíduos sólidos com alto potencial poluidor. A pirólise destaca-se como uma alternativa para estes resíduos, permitindo sua conversão em bio-óleo, char e gases não condensáveis. Este trabalho estudou os aspectos envolvidos na pirólise do resíduo de couro, utilizando um reator de leito fluidizado. $\mathrm{O}$ parâmetro variado nos ensaios foi a temperatura $(723 \mathrm{~K}$ e $873 \mathrm{~K})$ e a vazão de $\mathrm{N}_{2}\left(0,034 \mathrm{~m}^{3} / \mathrm{h}\right.$ e $\left.0,030 \mathrm{~m}^{3} / \mathrm{h}\right)$. A pirólise à temperatura de $873 \mathrm{~K}$ apresentou maior rendimento de bio-óleo. A caracterização do produto líquido evidenciou a presença de compostos orgânicos oxigenados e não oxigenados em sua composição. O char obtido foi caracterizado e constatou-se que o mesmo apresenta morfologia porosa e é composto basicamente por $\mathrm{C}, \mathrm{Cr}, \mathrm{Na}, \mathrm{Mg}$ e $\mathrm{Cl}$. Assim, a pirólise dos resíduos de couro apresenta-se como uma alternativa para o tratamento destes resíduos, bem como para a obtenção de produtos úteis.
\end{abstract}

\section{INTRODUÇÃO}

A indústria coureira destaca-se pela quantidade considerável de resíduos gerados (Kong et al., 2013). Dentre os resíduos sólidos, grande parte enquadra-se na classificação de Resíduos Classe I - perigosos (ABNT 10.004, 2004), devido a presença de sais de cromo utilizados no processo de curtimento.

O desenvolvimento de alternativas que permitam a reutilização do resíduo de couro ou sua conversão em produtos úteis é assunto de urgência, visto que leis ambientais cada vez mais severas pressionam as empresas quanto ao reaproveitamento dos resíduos gerados. Diversos autores têm estudado alternativas para a destinação dos resíduos da indústria coureira contendo cromo (Dettmer et al., 2010; Piccin et al., 2010).

Neste contexto, o processo termoquímico de pirólise apresenta-se como uma opção interessante. Este processo, que já vem sendo largamente aplicado a resíduos orgânicos, consiste no aquecimento da biomassa em atmosfera inerte com a formação de três produtos: char, bio-óleo e gases não condensáveis (Bridgwater, 2012).

O rendimento e a qualidade dos produtos obtidos são influenciados pelos parâmetros empregados no processo de pirólise, pela natureza da biomassa e ainda pela escolha adequada do reator pirolítico. Dentre as opções disponíveis, o processo de pirólise é bem sucedido em reatores de leito fluidizado porque estes possuem elevada transferência de calor e massa entre 
as fases, baixos gradientes de temperatura em toda a sua extensão e fácil coleta de produtos. Esta configuração ainda é destacada devido à facilidade na operação, versatilidade e custos atrativos de implantação.

O bio-óleo obtido a partir da pirólise de um resíduo é um líquido de coloração marrom escuro, odor característico de fumaça e é composto por uma complexa mistura de compostos oxigenados e de certa porcentagem de água (Bridgwater, 2012). Este produto pode ser utilizado como fonte de matéria-prima para a geração de produtos de maior valor agregado, como um fluido transportador de energia, em caldeiras e ainda, como combustível. Para esta última aplicação, o bio-óleo precisa ter as seguintes características melhoradas: altos teores de oxigênio e água, alta acidez ( $\mathrm{pH} \sim 2,5)$, menor poder calorífico superior (PCS) $(17 \mathrm{MJ} / \mathrm{kg}$ ) que o do óleo combustível (43 MJ/kg), alta viscosidade, corrosividade e instabilidade, podendo sofrer polimerização e condensação ao longo do tempo (Bridgwater, 2012; Gil et al., 2012).

O char é composto basicamente de carbono elementar com alguns átomos de hidrogênio ligados. Além disso, contém também grande parte dos compostos inorgânicos originalmente presentes na biomassa (Yilmaz et al., 2007). São três os métodos conhecidos para a disposição ou utilização do char obtido através da pirólise de um resíduo qualquer: combustível sólido, ativação para uso como adsorvente e deposição em aterros sanitários, pois o processo de pirólise permite a fixação dos metais pesados no interior da matriz carbonosa, reduzindo o risco de lixivação (Gil et al., 2012; Yilmaz et al., 2007; Kong et al., 2013).

O objetivo deste trabalho é estudar a produção de materiais úteis a partir de resíduos de couro utilizando o processo de pirólise em reator de leito fluidizado. Pretende-se ainda avaliar o rendimento do processo de pirólise e investigar o efeito da temperatura final de operação na distribuição dos produtos. Por fim, espera-se caracterizar o bio-óleo e o char.

\section{MATERIAIS E MÉTODOS}

\subsection{Caracterização do resíduo de couro}

O resíduo de couro utilizado para a execução deste trabalho é proveniente da operação de rebaixamento do couro e foi fornecido pela empresa Peles Pampa, localizada na cidade de Portão, RS. O resíduo foi moído e classificado granulometricamente. Para os ensaios utilizouse resíduo com um diâmetro de partícula $\left(\mathrm{d}_{\mathrm{p}}\right)$ de $0,45 \mathrm{~mm}$, previamente seco.

A caracterização do resíduo de couro foi realizada a fim de avaliar seu potencial no processo de pirólise. Para tanto, analisou-se o mesmo quanto à umidade (ABNT NBR 11029: 2001) e PCS (ASTM D 5865-12). A avaliação da estabilidade térmica do resíduo de couro foi conduzida em atmosfera de $\mathrm{N}_{2}$, sob um fluxo de $50 \mathrm{~mL} / \mathrm{min}$ a uma taxa de aquecimento de 20 $\mathrm{K} / \mathrm{min}$, utilizando balança termogravimétrica da marca Shimadzu TGA-50. As análises de microscopia eletrônica de varredura (MEV) foram realizadas em um equipamento da marca Shimadzu modelo SSX-550, com deposição de ouro sobre as amostras.

\subsection{Procedimento experimental}


Os experimentos de pirólise foram realizados em um reator de leito fluidizado de bancada, que opera no regime batelada. A Figura 1 (a) e (b) apresenta, respectivamente um esquema 3D e um desenho esquemático do interior do reator utilizado.
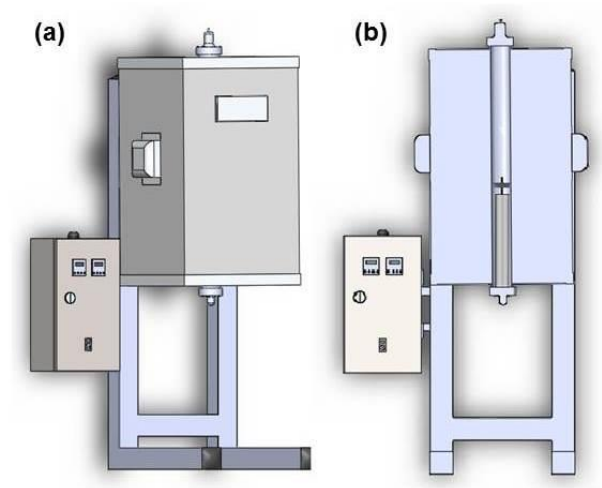

(c)

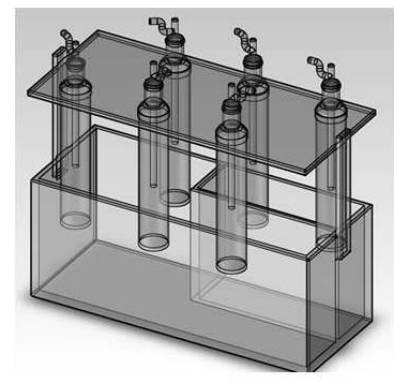

Figura 1 - (a) Desenho 3D do reator, (b) desenho esquemático do interior do equipamento e (c) desenho esquemático do sistema de coleta do bio-óleo.

Os parâmetros empregados nos ensaios de pirólise estão apresentados na Tabela 1. Os ensaios foram realizados em triplicata.

Coleta do bio-óleo: A coleta do bio-óleo foi conduzida segundo procedimento indicado pelo Comité Européén de Normalisation (CEN) (2004) em sua norma TC BT/TF 143 WI CSC 03002.4: 2004 adaptada. Utilizou-se uma sequência de 6 borbulhadores para a condensação do bio-óleo volatilizado durante a pirólise do resíduo de couro, conforme Figura 1 (c). Aos borbulhadores, com exceção do primeiro e do último, foram adicionados $40 \mathrm{~mL}$ de álcool isopropílico. Os mesmos ficaram imersos em um banho de gelo, sal e álcool isopropílico, cujo objetivo foi manter os borbulhadores a baixa temperatura.

Determinação das frações produzidas nos ensaios: A determinação da quantidade de char produzido foi realizada via pesagem do produto obtido ao final de cada experimento, após a secagem do mesmo em estufa, por aproximadamente 8 horas à temperatura de $375( \pm 2)$ K.

O volume de bio-óleo produzido foi quantificado a partir da evaporação da fração líquida obtida, composta pelo bio-óleo formado durante as reações de pirólise e pelo álcool isopropílico adicionado aos borbulhadores para a condensação dos gases pirolíticos. Para tanto, utilizou-se um evaporador rotativo à vácuo $(53,32 \mathrm{kPa}$ de pressão) modelo R110, da marca Brinkmann, ajustado a temperatura de $343 \mathrm{~K}$ através de um banho com água (Perry e Green, 1999). A fração líquida foi avolumada a $250 \mathrm{~mL}$ em um balão previamente seco e pesado e o processo foi iniciado. A massa do balão contendo bio-óleo foi determinada e, por diferença, a massa de bio-óleo obtido.

A fração de gás produzido foi estimada por balanço de massa.

\subsection{Caracterização dos produtos}


O char obtido através dos ensaios de pirólise de resíduo de couro foi caracterizado por MEV e ainda pela técnica de espectroscopia de dispersão em energia (EDS), realizada em um equipamento da marca Shimadzu modelo SSX-550, com deposição de ouro.

O bio-óleo foi caracterizado segundo a técnica de espectroscopia no infravermelho com transformada de Fourier (FTIR), conduzida no equipamento Nicolet IS10 - Thermo Scientific.

\section{RESULTADOS E DISCUSSÃO}

\subsection{Caracterização do resíduo de couro}

As amostras analisadas apresentaram umidade equivalente a 43,94\%, em base seca, e PCS igual a 48,65 kJ / kg. O resultado encontrado para o PCS do resíduo de couro vem ao encontro dos resultados obtidos por outros autores (Gil et al., 2012; Swarnalatha et al., 2008). Tal valor, aliado ao fato que o resíduo de couro tem origem animal, comprova sua classificação como biomassa e viabiliza o uso deste resíduo como fonte de energia.

As imagens obtidas através da análise de MEV do resíduo de couro antes do processo de pirólise são apresentadas na Figura 2 (a). Através da imagem de MEV é possível identificar a presença de uma rede de fibras no resíduo de couro (Pereira, 2006). A estrutura fibrosa observada é característica do colágeno, principal componente da pele original e o responsável por conferir estabilidade à pele (Gil et al., 2012).

As curvas de análise termogravimétrica (TGA) e da análise térmica diferencial (DTG), obtidas a partir da análise térmica do resíduo de couro, estão apresentadas na Figura 2 (b). Duas regiões principais de perda de massa podem ser observadas. A primeira região ocorre em uma temperatura próxima a $360 \mathrm{~K}$ e representa a perda de umidade livre e de outros compostos voláteis. A segunda região ocorre entre $600 \mathrm{~K}$ e $800 \mathrm{~K}$ e é atribuída à decomposição do resíduo de couro. Durante esta etapa ocorrem modificações na estrutura e na composição do resíduo, com a obtenção do char e a formação dos compostos condensáveis. Concomitantemente, ocorre a liberação de gases (Gil et al., 2012).
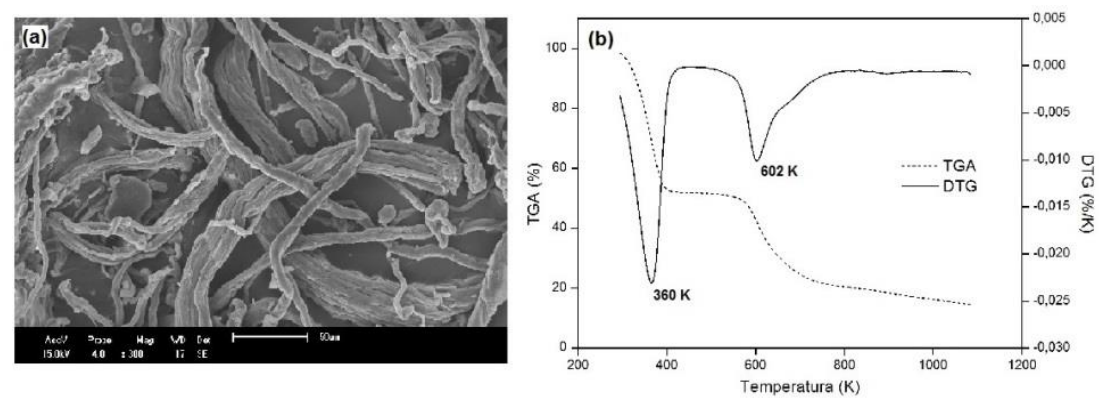

Figura 2 - Imagem de: (a) MEV e (b) TGA e DTG do resíduo de couro.

\subsection{Produtos de pirólise}

A Tabela 1 apresenta o rendimento dos produtos obtidos a partir dos ensaios de pirólise de resíduo de couro. 
Tabela 1 - Parâmetros utilizados e rendimento dos produtos obtidos nos ensaios de pirólise

\begin{tabular}{|c|c|c|c|c|c|}
\hline \multicolumn{3}{|c|}{ Parâmetros do ensaio de pirólise } & \multirow{2}{*}{$\begin{array}{c}\text { Char } \\
(\% \text { b.s. })^{*}\end{array}$} & \multirow{2}{*}{$\begin{array}{c}\text { Bio-óleo } \\
(\% \text { b.s. })^{*}\end{array}$} & \multirow{2}{*}{$\begin{array}{l}\text { Gás } \\
(\%)^{* *}\end{array}$} \\
\hline $\begin{array}{l}\text { Temperatura } \\
\text { (K) }\end{array}$ & $\begin{array}{c}\text { Taxa de aquecimento } \\
\qquad(\mathrm{K} / \mathrm{s})\end{array}$ & $\begin{array}{l}\text { Vazão de } \\
N_{2}\left(\mathbf{m}^{3} / h\right)\end{array}$ & & & \\
\hline 723 & 0,25 & 0,04 & 24,97 & 7,52 & 67,50 \\
\hline 873 & 0,4 & 0,03 & 20,92 & 11,29 & 67,79 \\
\hline
\end{tabular}

O rendimento dos produtos obtidos nos ensaios de pirólise apresentam divergências com relação ao determinado pela literatura, considerando o mesmo tipo de resíduo. Esta divergência pode ser atribuída a diferentes parâmetros utilizados para o processo de pirólise (Marcilla et al., 2012; Yilmaz et al., 2007).

Com relação à influência da temperatura na distribuição das frações de rendimento, observa-se que os valores obtidos estão de acordo com o indicado na literatura para um processo de pirólise convencional (Bridgwater, 2012). Conforme esperado, o rendimento de bio-óleo foi superior no ensaio de pirólise a $873 \mathrm{~K}$, indicando que, dentre as temperaturas testadas, esta favorece a formação de bio-óleo. Por outro lado, o ensaio de pirólise à temperatura menor (723 K) favorece a formação de char.

\subsection{Caracterização dos produtos}

Caracterização do char: A Figura 3 apresenta o MEV do char produzido durante o processo de pirólise do resíduo de couro, nas temperaturas de $723 \mathrm{~K}$ e $873 \mathrm{~K}$.
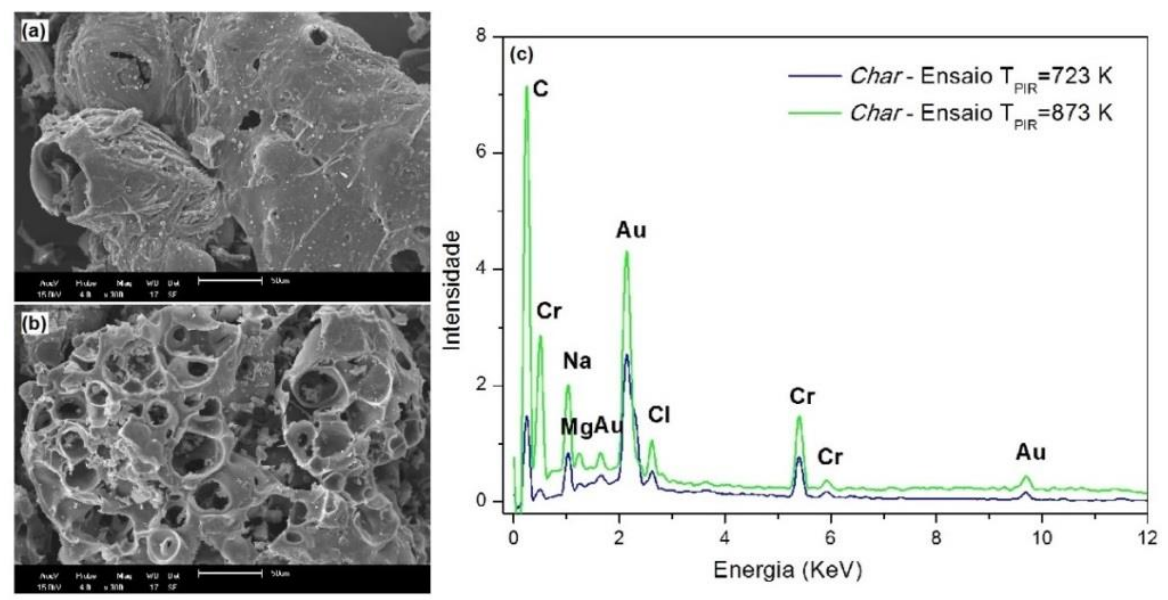

Figura 3 - MEV do char obtido através da pirólise do resíduo de couro: (a) temperatura de pirólise de $723 \mathrm{~K}$, aumento 300x; (b) temperatura de pirólise de $873 \mathrm{~K}$, aumento de 300x; (c) EDS do char obtido através da pirólise de resíduo de couro.

Conforme pode ser observado ao comparar a imagem de MEV do resíduo de couro com as imagens de MEV do char produzido, alterações morfológicas ocorrem durante o processo de pirólise. A análise de MEV evidencia que o char apresenta morfologia porosa, composta 
por poros de tamanhos variados e irregulares. A estrutura observada deve-se, possivelmente, à intensa eliminação de compostos voláteis durante as reações secundárias de pirólise (Sharma et al., 2001).

O char obtido no processo de pirólise a $723 \mathrm{~K}$ apresenta, além de poros, fibras em sua estrutura. A presença de fibras representa um indício de que, nesta temperatura, a pirólise do resíduo de couro ainda não foi completa. O resíduo de couro mantém a sua estabilidade térmica, possivelmente devido à estabilização causada pelo efeito do agente de curtimento sobre a estrutura do colágeno da pele (Gil et al., 2012). Por outro lado, nas imagens de MEV do char produzido a partir da pirólise a $873 \mathrm{~K}$ não são observadas estruturas fibrosas, evidenciando que a pirólise do resíduo de couro foi efetiva.

A análise de EDS do char obtido através dos ensaios de pirólise do resíduo de couro pode ser visualizada na Figura 3 (c). Segundo esta análise, o char oriundo do processo de pirólise do resíduo de couro é composto basicamente por $\mathrm{C}, \mathrm{Cr}, \mathrm{Na}, \mathrm{Mg}$ e $\mathrm{Cl}$. O elemento $\mathrm{Au}$, também presente no EDS do char, pode ser atribuído ao processo de deposição de ouro pelo qual o produto foi submetido a fim de possibilitar esta análise.

A composição do char apontada pela análise de EDS está de acordo com a literatura, que afirma que este produto é composto basicamente por carbono elementar e por grande parte dos compostos inorgânicos originalmente presentes na biomassa (Gil et al., 2012; Yilmaz et al., 2007).

Conforme pode ainda ser observado na Figura 3 (c), a composição do char não é alterada pela variação na temperatura final de pirólise. No entanto, uma pequena diferença na intensidade dos elementos é constatada. Esta divergência pode ser atribuída à incompleta decomposição do resíduo de couro na temperatura de $723 \mathrm{~K}$.

Caracterização do bio-óleo: A Figura 4 apresenta o resultado do FTIR do bio-óleo produzido a partir dos ensaios de pirólise do resíduo de couro. Para estes ensaios, utilizou-se o bio-óleo obtido após a evaporação no rotavapor à vácuo.

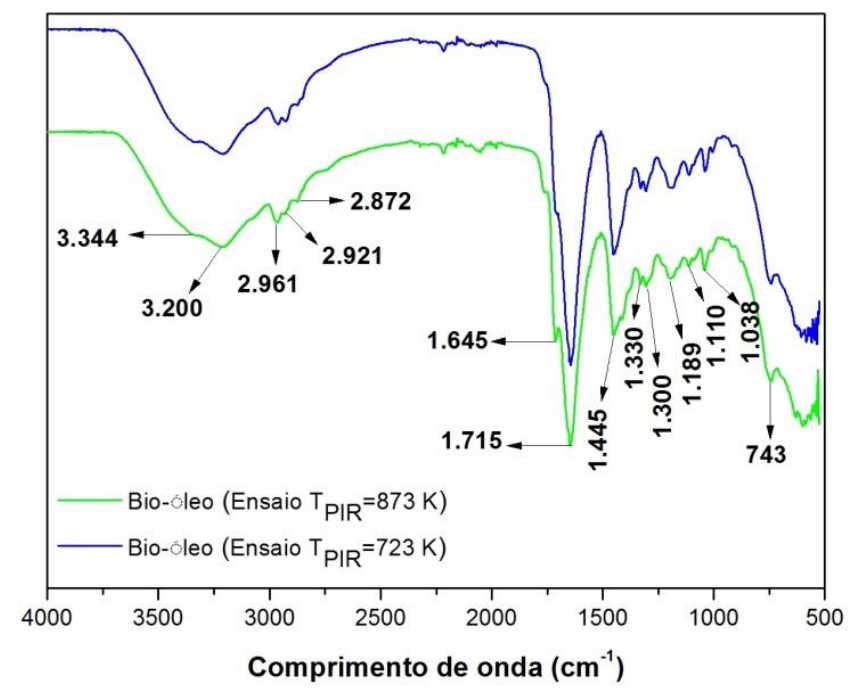

Figura 4 - FTIR do bio-óleo: temperaturas de 723 K e 873 K. 
Os espectros obtidos, tanto para o bio-óleo produzido a partir dos experimentos de pirólise na temperatura de $723 \mathrm{~K}$ quanto para os ensaios na temperatura de $873 \mathrm{~K}$ estão de acordo com os valores observados na literatura, uma vez que apresentam semelhança na distribuição dos grupos funcionais.

Os espectros revelam uma banda na região de $3.344 \mathrm{~cm}^{-1}$ e outra na região de $3.200 \mathrm{~cm}^{-}$ 1, ambas caracterizam a ligação O-H dos grupos álcool, fenol, ou ácido carboxílico (Onay, 2007; Ozbay et al., 2008). As bandas $2.961 \mathrm{~cm}^{-1}, 2.921 \mathrm{~cm}^{-1}$ e $2.872 \mathrm{~cm}^{-1}$ indicam a presença dos grupos funcionais $\mathrm{CH}_{3}$ (para as duas primeiras bandas) e $\mathrm{CH}_{2}$ (para a terceira banda) (Zhang et al., 2012). O comprimento de onda em aproximadamente $1.645 \mathrm{~cm}^{-1}$ é típico de ligações $\mathrm{C}=\mathrm{C}$ (Ozbay et al., 2008). A banda em aproximadamente $1.715 \mathrm{~cm}^{-1}$ corresponde ao grupo carbonila $(\mathrm{C}=\mathrm{O})$ e indica a presença de cetonas e aldeídos. As bandas de $1.330 \mathrm{~cm}^{-1} \mathrm{e}$ $1.445 \mathrm{~cm}^{-1}$ são peculiares às ligações C-H (Onay, 2007; Ozbay et al., 2008). As bandas 1.300 $\mathrm{cm}^{-1}, 1.189 \mathrm{~cm}^{-1}, 1.110 \mathrm{~cm}^{-1}$ e $1.038 \mathrm{~cm}^{-1}$ caracterizam as ligações $\mathrm{O}-\mathrm{H}$ e C-O, indicando a presença de fenóis, álcoois e ésteres. O pico que aparece em $743 \mathrm{~cm}^{-1}$ é característico das ligações $\mathrm{C}$-H fora do plano, comprovando a presença de compostos aromáticos no bio-óleo analisado (Onay, 2007).

Eventualmente, o bio-óleo pode conter resquícios de Cr (III) em sua composição, análises para determinação deste metal estão em andamento.

\section{CONCLUSÃO}

A partir dos resultados dos ensaios de pirólise de resíduos de couro apresentados neste trabalho, pode-se verificar que a maior produção de bio-óleo ocorre nos ensaios à temperatura de 873 K. Para o char, a maior produção observada foi nos ensaios à temperatura de $723 \mathrm{~K}$.

A análise de FTIR do bio-óleo mostrou a presença dos grupos funcionais $\mathrm{O}-\mathrm{H}, \mathrm{C}-\mathrm{H}$, $\mathrm{C}=\mathrm{C}, \mathrm{C}-\mathrm{O}$, evidenciando que este produto apresenta, em sua composição, álcoois, fenóis e cetonas. $\mathrm{O}$ char produzido apresentou morfologia porosa e composição composta por $\mathrm{C}, \mathrm{Cr}$, $\mathrm{Na}, \mathrm{Mg}$ e $\mathrm{Cl}$, conforme evidenciado através das análises de MEV e EDS.

Os resultados apresentados por este trabalho apontam a possibilidade da utilização do processo de pirólise em leito fluidizado para o tratamento dos resíduos gerados pela indústria coureira.

\section{REFERENCIAS}

AMERICAN SOCIETY FOR TESTING AND MATERIALS. ASTM D 5865 - Standard Test Method for Gross Calorific Value of Coal and Coke. Pennsylvania, 2012.

ASSOCIAÇÃO BRASILEIRA DE NORMAS TÉCNICAS. NBR 10.004: Resíduos sólidos Classificação. Rio de Janeiro, 2004.

ASSOCIAÇÃO BRASILEIRA DE NORMAS TÉCNICAS. NBR 11.029: Peles e couros Determinação do teor de matéria volátil. Rio de Janeiro, 2001.,

BRIDGWATER, A. Review of fast pyrolysis of biomass and product upgrading. Biomass and Bioenergy, v. 38, p. 68-94, 2012. 
COMITÉ EUROPÉÉN DE NORMALISATION. CEN BT/TF 143: Biomass Gasification: Tar and Particles in Product Gases - Sampling and Analysis. Bruxelas, 2004.

DETTMER, A. ; MARCÍLIO, N. R.; MARILIZ, G. S.; NUNES, K. G. P. Tanning Using Basic Chrome Sulfate Obtained from Ash Produced in the Thermal Treatment of Leather Wastes. J. Am. Leather Chem. Assoc., v. 105, p. 280-288, 2010.

GIL, R.; GIRÓN, R.; LOZANO, M.; RUIZ, B.; FUENTE, E. Pyrolysis of biocollagenic wastes of vegetable tanning. Optimization and kinetic study. J. Anal. Applied Pyrol., v. 98, p. 129-136, 2012.

KONG, J.; YUE, Q.; HUANG, L.; GAO, Y.; SUN, Y.; GAO, B.; LI, Q.; WANG, Y. Preparation, characterization and evaluation of adsorptive properties of leather waste based activated carbon via physical and chemical activation. Chem. Eng. J., v. 221, p. 62-71, 2013.

ONAY, O. Fast and catalytic pyrolysis of pistacia khinjuk seed in a well-swept fixed bed reactor. Fuel, v. 86, p. 1452-1460, 2007.

ÖZBAY, N.; APAYDIN-VAROL, E.; UZUM, B.; PUTUN, A. Characterization of bio-oil obtained from fruit pulp pyrolysis. Energy, v. 33, p. 1233- 1240, 2008.

PEREIRA, S. Obtenção de cromato de sódio a partir das cinzas de incineração de resíduos de couro do setor calçadista visando à produção de sulfato básico de cromo. 2006. $122 \mathrm{f}$. Dissertação (Mestrado em Engenharia Química) - Escola de Engenharia, Universidade Federal do Rio Grande do Sul, Porto Alegre, 2006.

PERRY, R.; GREEN, D. Perry's chemical engineers' handbook. New York: McGraw-Hill, 1999.

PICCIN, J. S.; GOMES, C. S.; FERIS, L. A.; GUTTERRES, M.. Kinetics and isotherms of leather dye adsorption by tannery solid waste. Chem. Eng. J., v. 183, p. 30-38, 2010

SHARMA, R.; WOOTEN, J.; BALIGA, V.; HAJALIGOL, M. Characterization of chars from biomass-derived materials: pectin chars. Fuel, v. 80, p. 1825-1836, 2001.

SWARNALATHA, S.; SRINIVASULU, T.; SRIMIRALI, M.; SEKARAN, G. Safe disposal of toxic chrome buffing dust generated from leather industries. J. Hazard. Mater., v. 150, p. 290-299, 2008.

YILMAZ, O.; KANTARLI, I; YUKSEL, M.; SAGLAM, M.; YANIK, J. Conversion of leather wastes to useful products. Resour. Conserv. Recycl., v. 49, p. 436-448, 2007.

ZHANG, Y.; CHEN, D.; ZHANG, D.; ZHU, X. TG-FTIR analysis of bio-oil and its pyrolysis/gasification property. J. Fuel Chem. Technol., v. 40, p. 1194-1199, 2012. 